\title{
Limited mediastinal lymph node dissection for non-small cell lung cancer according to intraoperative histologic examinations
}

Tatsuya Yoshimasu, MD, PhD, ${ }^{\text {a }}$ Shinichiro Miyoshi, MD, PhD, ${ }^{\text {b }}$ Shoji Oura, MD, PhD, ${ }^{\text {a }}$ Issei Hirai, MD, ${ }^{\text {a }}$ Yozo Kokawa, MD, and Yoshitaka Okamura, MD, PhDa

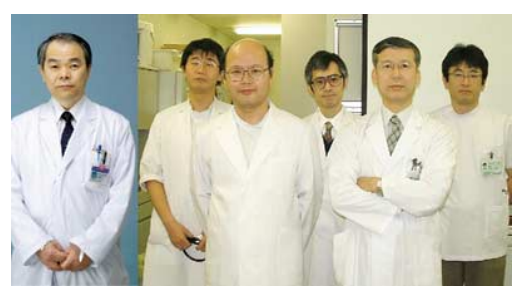

Miyoshi, Kokawa, Oura, Yoshimasu, Okamura, Hirai (left to right)

See related editorial on page 241.
Background: Although radioisotopic procedures are commonly used to detect sentinel lymph nodes in breast cancer surgery, these procedures are often problematic and not necessarily suitable for lung cancer surgery.

Methods: Our previous study revealed that the mediastinal sentinel lymph node, defined as the regional mediastinal lymph node, consisted of nodes 2,3 , or 4 in right upper lobe cancers; 3,7 , or 8 in right lower lobe cancers; 4,5 , or 7 in left upper lobe cancers; and 4, 7, or 8 in left lower lobe cancers. On the basis of these findings, we pathologically investigated one representative lymph node at each of the 3 levels dissected during surgical intervention in 69 patients with non-small cell lung cancer from September 1993 through December 2002. Fifty-eight patients with lung cancer underwent lobectomies with limited mediastinal lymph node dissection according to this strategy.

Results: Mediastinal lymph node recurrence was observed in only one patient during $41 \pm 25$ months (maximum, 98 months) of follow-up. The cancer-specific 5-year survivals were $96.6 \%$ in patients with pathologic stage IA disease $(\mathrm{n}=31)$ and $67.4 \%$ in patients with stage IB disease $(n=16)$.

Conclusion: These results suggested that limited mediastinal lymph node dissection is applicable to patients with non-small cell lung cancer whose regional mediastinal lymph nodes are not metastatic.

$\mathrm{S}$ entinel lymph node mapping techniques have been extensively studied in melanoma and breast cancers, ${ }^{1-3}$ and they are now applied to most solid tumors. Usually these techniques involve the preoperative injection of a blue dye or radioisotope into the peritumoral tissue, which allows the sentinel lymph nodes to be detected during surgical intervention. However, there are some difficulties with these methods in their application to lung cancer surgery. First, preoperative computed tomography-guided injections of a radioisotope or blue dye into the lung are somewhat troublesome; second, blue dye is not so beneficial for anthracotic lymph nodes.

$\mathrm{We}^{4}$ previously investigated which mediastinal lymph node levels should be examined during surgical intervention to diagnose N2 or less than N2 disease in patients with bronchogenic carcinomas. With primary tumors, 3 mediastinal lymph node levels were selected for each lobe and defined as regional mediastinal lymph nodes; the idea of the regional mediastinal lymph node is quite similar to that of the sentinel lymph node. However, investigation of the regional mediastinal lymph node is more practical and convenient than sentinel lymph node examination by means of tracer-guided sentinel lymph node biopsy techniques because regional mediastinal lymph nodes are examined pathologically during surgical intervention without any special equipment. We suggested that if the regional mediastinal lymph nodes are 
TABLE 1. Lymph node levels for regional and standard mediastinal node dissection

\begin{tabular}{lcl}
\hline Tumor location & $\begin{array}{c}\text { Regional node } \\
\text { dissection }\end{array}$ & $\begin{array}{c}\text { Standard node } \\
\text { dissection }\end{array}$ \\
\hline Right upper lobe & $2,3,4$ & $1,2,3,4,7$ \\
Right lower lobe & $3,7,8$ & $1,2,3,4,7,8,9$ \\
Left upper lobe & $4,5,7$ & $4,5,6,7$ \\
Left lower lobe & $4,7,8$ & $4,5,6,7,8,9$ \\
\hline
\end{tabular}

\section{TABLE 2. Patient characteristics}

\begin{tabular}{lc}
\hline Mean age (y) & $68 \pm 8$ (44-83) \\
Sex & 45 \\
$\quad$ Male & 13 \\
$\quad$ Female & \\
Primary site & 24 \\
$\quad$ RUL & 10 \\
RLL & 13 \\
LUL & 11 \\
LLL & \\
Histology & 24 \\
$\quad$ Adenocarcinoma & 29 \\
Squamous cell carcinoma & 2 \\
Large cell carcinoma & 2 \\
Carcinoid & 1 \\
Unknown & \\
Surgical intervention & 58 \\
Lobectomy & 1 \\
Pneumonectomy & \\
\hline
\end{tabular}

$R U L$, Right upper lobe; $R L L$, right lower lobe; $L U L$, left upper lobe; $L L L$, left lower lobe.

not metastatic, dissection of mediastinal lymph nodes could be stopped at this point without the additional mediastinal lymph node dissection.

Thus, a prospective study of limited mediastinal lymph node dissection on the basis of the idea of the regional mediastinal lymph node was commenced in 1993. This report shows the follow-up results of this study and discusses the validity of the proposed method.

\section{Patients and Methods}

As a preliminary study, ${ }^{4}$ we retrospectively reviewed the patterns of mediastinal lymph node metastasis in relation to the location of primary lung cancer in 55 patients with pathologic $\mathrm{N} 2$ disease who underwent resection of a primary lesion and standard mediastinal lymphadenectomy. Lymph node mapping was conducted according to the criteria of the Japanese Lung Cancer Society. ${ }^{5}$ Mediastinal lymph node metastasis was detected in $100 \%$ (17/17) of patients with right upper lobe cancers by examination of node levels 2, 3, and 4; in 91\% (20/22) by examination of node levels 3 , 7 , and 8 in right lower lobe cancers; in $91 \%(10 / 11)$ by examination of node levels 4,5 , and 7 in left upper lobe cancers; and in $100 \%(5 / 5)$ by examination of node levels 4,7 , and 8 in left lower
TABLE 3. Postoperative staging and LN status

\begin{tabular}{lr}
\hline Pathologic stage & \\
IA & 31 \\
IB & 16 \\
IIA & 1 \\
IIB & 7 \\
IIIA & 2 \\
IIIB & 1 \\
IV & 0 \\
LN status & \\
N0 & 52 \\
N1 & 5 \\
N2 & 1 \\
N3 & 0
\end{tabular}

$L N$, Lymph node.

\section{TABLE 4. Recurrence}

\begin{tabular}{ll}
\hline Distant metastasis & 7 \\
Local recurrence & 2 \\
Bronchial stump & 1 \\
Mediastinal LN & 1
\end{tabular}

$L N$, Lymph node.

lobe cancers. Node levels were not selected for the middle lobe because of the small number. As a prospective study, these 3 levels were investigated pathologically during surgical intervention by examining one representative lymph node most strongly suspected to be metastatic, which means the largest node, nonanthracotic node, or both in each of the 3 levels dissected in 61 patients. The $\mathrm{N}$ status diagnosed according to the frozen sections (f-N) during surgical intervention was compared with the $\mathrm{N}$ status diagnosed according to the permanent sections ( $\mathrm{p}-\mathrm{N}$ ) obtained after the standard mediastinal dissections. The f-N value was consistent with the $\mathrm{p}-\mathrm{N}$ value in 39 patients with $\mathrm{N} 0$ or $\mathrm{N} 1$ disease and in 16 patients with $\mathrm{N} 2$ disease or more. Thus, $90 \%$ of the $\mathrm{f}-\mathrm{N}$ values were consistent with the $\mathrm{p}-\mathrm{N}$ values. The $\mathrm{f}-\mathrm{N}$ values resulted in underdiagnosis in 6 patients, 4 of whom had a micrometastasis in only one of the lymph nodes dissected from the 3 levels. Because the 3 levels were completely dissected, there were no residual metastatic lymph nodes. The remaining 2 patients had metastatic lymph nodes outside the 3 levels. Standard mediastinal lymph node dissection was performed for these 2 patients because the lymph node was macroscopically suspected to be metastatic; this was pathologically confirmed by means of frozen sectioning during surgical intervention. The 3 levels for each lobe are shown in Table 1.

The present study was conducted on the basis of the results of the preliminary study. From September 1993 through December 2002, 308 patients underwent surgical intervention for primary lung cancer in our institute. Patients with preoperative mediastinoscopy, patients with $\mathrm{N} 2$ disease proved by video-assisted thoracic surgery, and those who had undergone partial resections or segmentectomies were excluded. Sixty-nine patients from whom informed consent for limited mediastinal lymph node dissection was obtained were included in this study. A total of 3 lymph nodes (one representative mediastinal lymph node in each level) were 


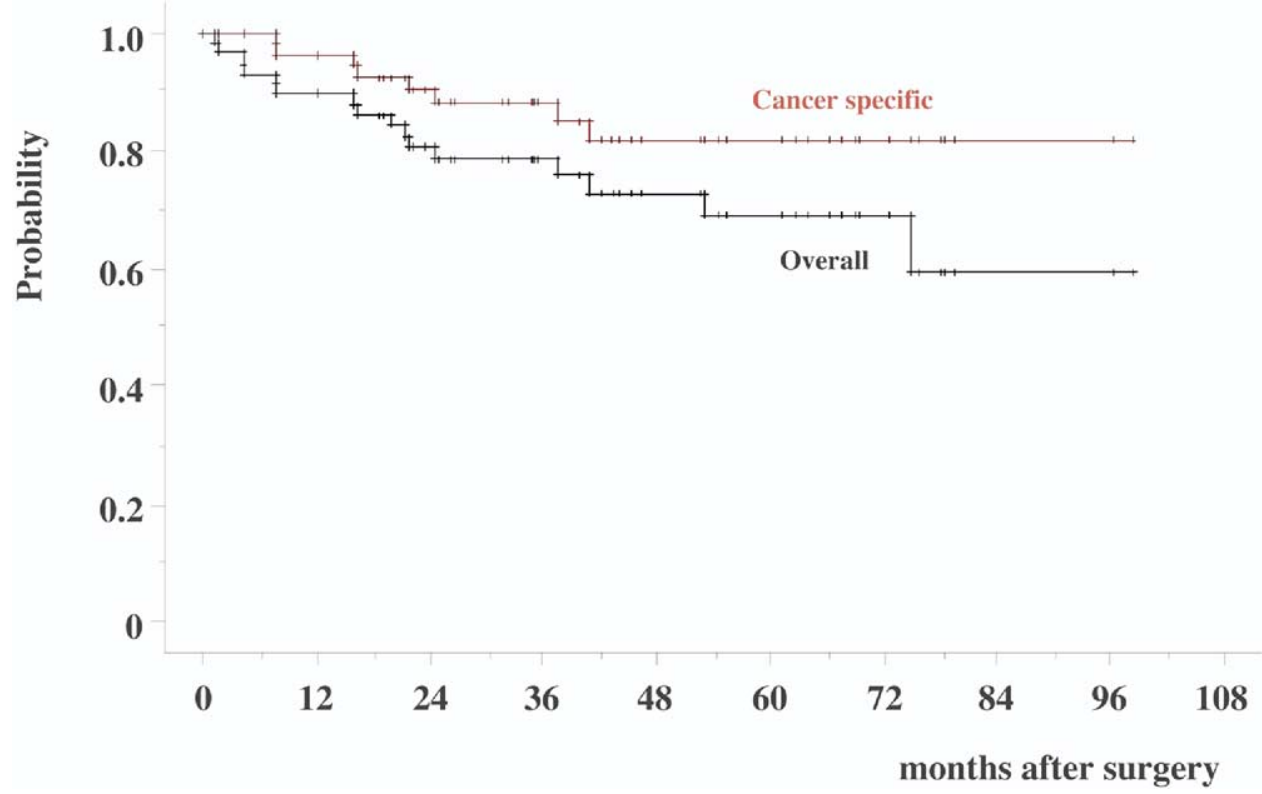

Figure 1. Cancer-specific and overall survivals of the 58 patients who underwent limited mediastinal lymph node dissection.

examined by means of frozen sectioning immediately after completing dissections of the hilar lymph nodes and regional mediastinal lymph nodes. If the frozen sections did not show lymph node metastasis, no further lymph node dissection was performed. If the frozen sections revealed lymph node metastasis, standard mediastinal lymph node dissection was conducted. All of the dissected lymph nodes, including the frozen sections, were examined by means of permanent sectioning. The lymph node levels of the limited (regional) and standard mediastinal node dissections are shown in Table 1.

The patients were followed up every month within the first 6 months after surgical intervention at an outpatient clinic and every 2 months thereafter. During the follow-up period, plain chest $\mathrm{x}$-ray films and serum tumor marker levels were obtained. Brain, chest,

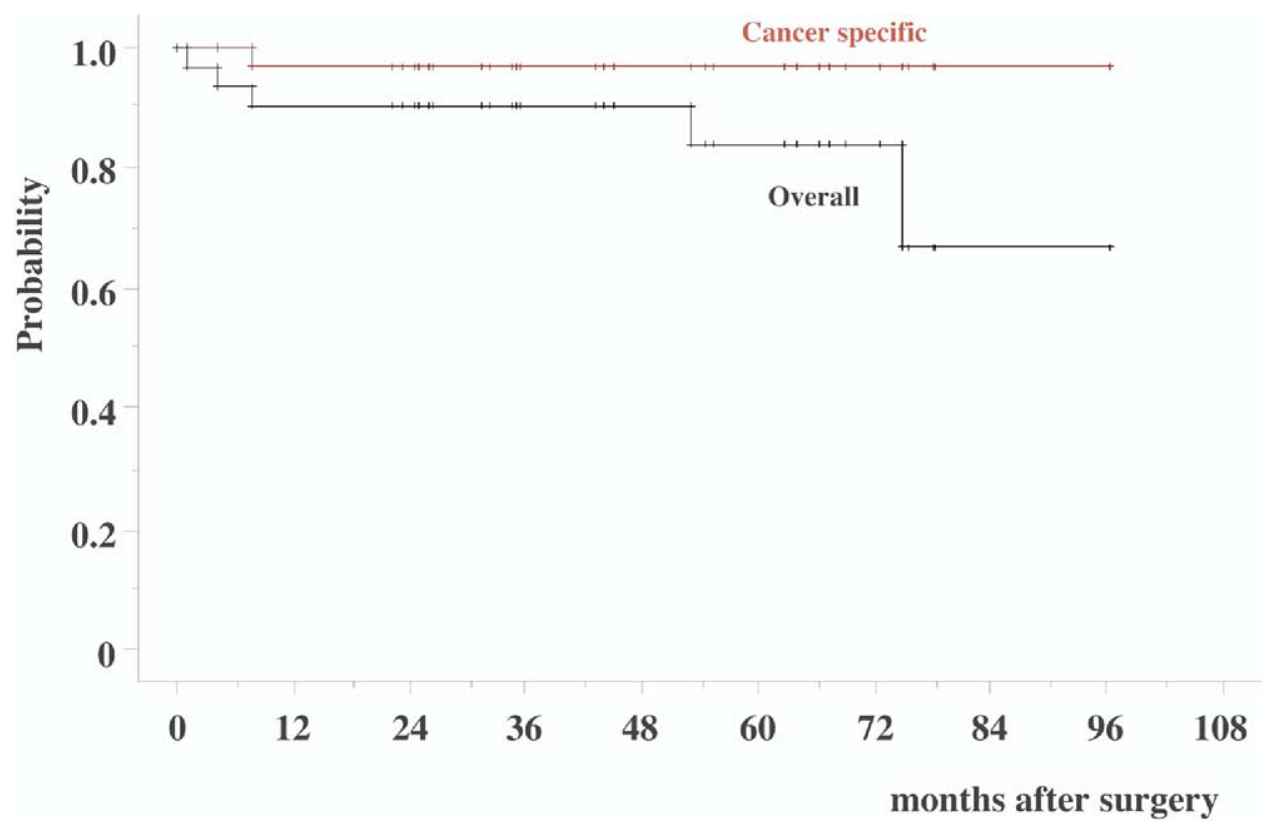

Figure 2. Cancer-specific and overall survivals of the 31 patients with stage la disease. 


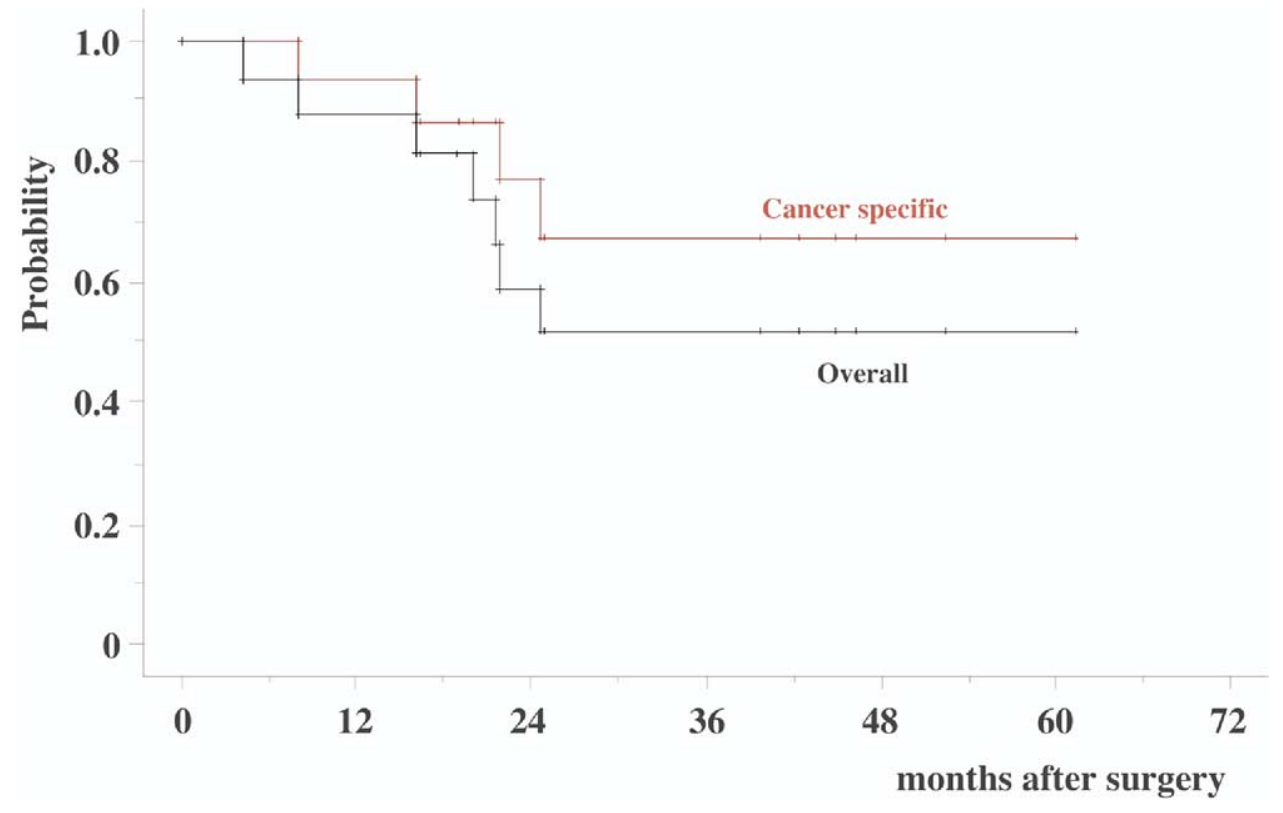

Figure 3. Cancer-specific and overall survivals of the 16 patients with stage lb disease.

and abdominal computed tomographic scans, as well as bone scintigraphies, were performed every 6 months to detect tumor recurrence. The mean follow-up time of the patients was $41 \pm 25$ months (range, 2-98 months).

All values are given as the mean \pm standard deviation. The Kaplan-Meier method was used to evaluate patient prognosis.

\section{Results}

Of the 69 cases studied, 11 were positive for regional mediastinal lymph nodes, and these patients underwent standard mediastinal lymph node dissections. Fifty-eight were negative for regional mediastinal lymph nodes, and these patients did not undergo additional mediastinal lymph node dissection. The characteristics of the 58 patients are shown in Table 2 .

Table 3 shows the pathologic staging and lymph node status of the 58 patients studied. Most patients were classified as having stage Ia or Ib disease. Postoperative staging revealed 1 false-negative case; micrometastasis was not detected in the frozen section from this patient. During the follow-up period, there were 9 recurrent cases, including 7 distant and 2 local recurrences (Table 4). Only one mediastinal lymph node recurrence was observed outside of the 3 levels.

The Kaplan-Meier survival curves of the 58 patients are shown in Figure 1. The cancer-specific 5-year and overall 5 -year survivals were $82.0 \%$ and $69.2 \%$, respectively. The survival curves of the patients with stage Ia disease $(\mathrm{n}=31)$ are shown in Figure 2; their cancer-specific and overall 5 -year survivals were $96.6 \%$ and $83.9 \%$, respectively. The survival curves of the patients with stage $\mathrm{Ib}$ disease $(\mathrm{n}=16)$ are shown in Figure 3; their cancer-specific and overall 5 -year survivals were $67.4 \%$ and $51.7 \%$, respectively. Good prognoses were observed, equal to those of the latest major report in Japan on 7508 patients with lung cancer. ${ }^{6}$

\section{Discussion}

Lymph node metastasis is the most important prognostic factor of localized non-small cell lung cancer (NSCLC). ${ }^{7-9}$ Accurate detection of lymph node metastasis therefore yields accurate predictions of patient prognosis. Lobectomy and standard mediastinal node dissection are common surgical procedures for localized NSCLCs in Japan. ${ }^{5}$ Hilar and mediastinal node dissections might improve survival in patients with stage II and IIIa NSCLC ${ }^{10}$; however, it cannot be suggested that lymph node dissection improves the prognosis of patients without lymph node metastasis. To diagnose N1 or lower diseases, mediastinal lymph nodes must be investigated because skip metastasis to mediastinal lymph nodes is often observed. ${ }^{11}$

Sentinel lymph node-mapping procedures have already been applied to most solid malignant tumors. ${ }^{1-3,12}$ In breast cancers sentinel lymph node-mapping procedures are becoming the standard operative procedure. Liptay and associates $^{13}$ reported that radioisotope (RI)-guided sentinel lymph node mapping techniques are applicable for lung cancer surgery. It has also been reported that precise histologic examinations of sentinel lymph nodes yield more accurate diagnoses of lymph node metastasis. Schmidt and 
colleagues ${ }^{14}$ revealed the accuracy of RI-guided sentinel lymph node mapping techniques in lung cancer. However, the validity of sentinel lymph node mapping techniques for limited mediastinal lymph node dissection has yet to be determined.

The 3 determined levels in the mediastinal lymph nodes (Table 1) were recognized as the regional mediastinal lymph nodes for each lobe. The 3 levels are extremely consistent with the anatomic lymphatic efferent pathways from each lung. ${ }^{15,16}$ The patterns of mediastinal lymph node metastasis reported in other reports are relatively similar to our results, ${ }^{11,16,17}$ and therefore the idea of determining and examining 3 levels of mediastinal lymph nodes during surgical intervention is similar to the method of sentinel lymph node mapping. However, our method seems more practical and convenient in the clinical setting than sentinel lymph node mapping, which requires special equipment.

Table 1 compares the settings of limited (regional) mediastinal lymph node dissection and standard lymph node dissection. Limited (regional) mediastinal lymph node dissection is apparently less invasive compared with standard lymph node dissection, except for the left upper lobe.

Residual mediastinal lymph node recurrence was experienced in one case with an adenocarcinoma in the left lower lobe and partial involvement of the left upper lobe. The patient underwent a left lower lobectomy and partial resection of the left upper lobe with limited (regional) mediastinal dissection of lymph nodes 4,7 , and 8 . Recurrence in lymph nodes $1,2,3$, and 5 was observed 13 months after surgical intervention. It is likely that we failed to detect the lymph node metastasis in level 5 , which is the regional lymph node of the left upper lobe. Therefore, our method should not be applied to patients with extralobar involvement.

Rare cases with extraregional skip metastasis have been reported previously (ie, metastasis to the level 7 nodes in upper lobe cancers). Anatomic investigation, including lymph scintigraphy, revealed that direct lymphatic flow to extraregional lymph nodes existed in some patients. ${ }^{11,15}$ Although there was no extraregional nodal recurrence in our patients, we surmise that the small possibility of extraregional skip metastasis exists in the limited mediastinal lymph node dissection.

The survivals of the patients who underwent limited (regional) mediastinal lymph node dissection showed no inferiority to those in the latest report in Japan on lung cancer. ${ }^{6}$ There was no mediastinal lymph node recurrence in the 57 patients whose indications were thought to be appropriate. These findings indicate the applicability and validity of limited mediastinal lymph node dissection in selected patients with NSCLC according to intraoperative histologic examinations.

From 1999, a prospective randomized trial of nodal sampling versus complete mediastinal nodal dissection was conducted by the American College of Surgeons Oncology Group (ACOSOG), and it was recently closed. This study consisted of 1000 patients (one arm $=500$ patients). It is expected that the follow-up results will confirm the validity of the nodal sampling method.

\section{References}

1. Morton DL, Duan Ren W, Wong SG, et al. Technical details of intraoperative lymphatic mapping for early stage melanoma. Arch Surg. 1992;127:392-9.

2. Giuliano AE, Kirgan DM, Guentha JM, Morton DL. Lymphatic mapping and sentinel lymphadenectomy for breast cancer. Ann Surg. 1994;220:391-401.

3. Giuliano AE, Dale PS, Turner RR, Morton DL, Evans SW, Kranse DL. Improved axillary staging of breast cancer with sentinel lymphadenectomy. Ann Surg. 1995;222:394-5.

4. Miyoshi S, Maebeya S, Suzuma T, Bessho T, Hirai I, Tanino H, et al. Which mediastinal lymph node should be examined during operation for diagnosing N0 or N1 disease in bronchogenic carcinoma? [in Japanese]. Jpn J Lung Cancer. 1997;37:475-84.

5. The Japan Lung Cancer Society. General rule for clinical and pathological record for lung cancer. Tokyo: Kimpodo; 2003.

6. Shirakusa T, Kobayashi K. Lung cancer in Japan. Analysis of lung cancer registry for resected cases in 1994. Japanese Joint Committee of Lung Cancer Registry. J Jpn Assoc Chest Surg. 2002;16:757-68.

7. Naruke T, Suemasu K, Ishikawa S. Lymph node mapping and curability at various levels of metastasis in resected lung cancer. $J$ Thorac Cardiovasc Surg. 1978;76:832-9.

8. Mountain CF, Dresler CM. Regional lymph node classification for lung cancer staging. Chest. 1997;111:1718-23.

9. Riquet M, Manac'h D, Pimpec-Barthes F, et al. Prognostic significance of surgical-pathologic N1 disease in non-small cell carcinoma of the lung. Ann Thorac Surg. 1999;67:1572-6.

10. Keller SM, Adak S, Wagner H, Johnson DH. Mediastinal lymph node dissection improves survival in patients with stages II and IIIa nonsmall cell lung cancer. Eastern Cooperative Oncology Group. Ann Thorac Surg. 2000;70:358-65.

11. Watanabe Y, Shimizu J, Tsubota M, Iwa T. Mediastinal spread of metastatic lymph nodes in bronchogenic carcinoma. Chest. 1990;97: 1059-65.

12. Wood TF, Tsioulias GJ, Morton DL, et al. Focused examination of sentinel lymph nodes upstages early colorectal carcinoma. Am Surg. 2000;66:998-1003.

13. Liptay MJ, Grondin SC, Fry WA, Pozdol C, Carson D, Knop C, et al. Intraoperative sentinel lymph node mapping in non-small cell lung cancer improves detection of micrometastases. J Clin Oncol. 2002;20: 1984-8.

14. Schmidt FE, Woltering EA, Webb WR, Garcia OM, Cohen JE, Rozans MH. Sentinel nodal assessment in patients with carcinoma of the lung. Ann Thorac Surg. 2002;74:870-5.

15. Okada Y. Lymphatic drainage route of the lung; lung cancer and pulmonary lymphatic system [in Japanese]. Tokyo: Kimpodo; 1989.

16. Libshitz HI, Mckenna RJ, Mountain CF. Patterns of mediastinal metastases in bronchogenic carcinoma. Chest. 1986;90:229-32.

17. Nohl HC. The spread of carcinoma of the bronchus. London: LloydLuke Ltd; 1962. p. 17-44. 